\title{
Numerical Simulation of Winglet to Optimization Design
}

\author{
Liu Xiaolei ${ }^{\mathrm{a}}$, Song Limin ${ }^{\mathrm{b}}$, Jin Dun $^{\mathrm{c}}$, Hu Tieyu ${ }^{\mathrm{a}}$ and Li song ${ }^{\mathrm{a}}$ \\ Aviation University of Air Force, Changchun, Jilin, China \\ aliuxiaolei@126.com, ' liminsong_2001@163.com, jindun@sina.com
}

Keywords: winglet; optimum design; numerical simulation; drag reduction

\begin{abstract}
Test flight results show that, the induced drag reduces $15 \%$ that the plane tototal resistance will decrease $6.5 \%$ and the range will increase $7.5 \%$. At present, new research and development aircraft, such as the Boeing787 "dream" aircraft and some airliners are in operation (Airbus A330, A340, Boeing737-800ER) and so on, have adopted the winglet. The drag reduction effect of winglet has been obviously. Because the drag reduction effect of winglet are restricted by many factors, such as the inclination angle, installation angle, winglets airfoil, winglet height, alula area and root tip ratio and so on. So winglet drag reduction effect in different plane are not the same which can be seen from the domestic and foreign literature. A winglet with more ideal drag reduction effect is an actively explore the topic for the global aircraft designers in recent years. In this paper, the large number of documents and materials on home and abroad are referenced. And the winglet is designed by universal CFD numerical simulation calculation method to increase the drag reduction effect.
\end{abstract}

\section{Introduction}

Modern aircraft design is fine multidisciplinary fusion and design optimization technology. It is highly integrated, especially aerodynamic layout design innovation. And the wing aero dynamic design is one of the core technologies for the plane layout design. The use of supercritical airfoil and improving the aerodynamic efficiency is an important aspect of the design of airfoil. The fusion winglet can hinder effectively airflow on the wing spanwise to formate the vortex. The vortex structure can be damaged, induced drag can be reduced thereby reducing the resistance, the aerodynamic efficiency can be improve, and the consumption rate of transport fuel also can be reduced. It is has become a model for fine design in recent years [1] [2].

The winglet during the course of civil aircraft application and development has been widespread concern for domestic and pneumatic design experts [3]. The earliest winglets is he winglet by NASA Langley of Whitcomb research and development center in twentieth Century, and it is installed in the KC2135 filling machine.

With the deterioration of the energy shortage and people's increasing concern about environmental issue, efficient, energy-saving, environmental friendly mode of economic development has become the major theme of the world economic development in the contemporary era[4]. Airplane is a kind of efficient and fast means of transportation, it need to consume a large amount of fuel. Therefore, how to reduce fuel consumption and improve flight efficiency is increasingly concerned by the aircraft designers. The key of achieving this goal is to reduce the drag and increase the lift. Particularly, reducing the drag in flight is an important aspect to improve the characteristics of a large transport airplane. The drag of large-scale long-range transport airplane is mainly consist of induced drag, pressure drag and friction drag. It is noteworthy that the induced drag accounts for about $40 \%$ of the total drag during cruising flight, so reducing the induced drag is an important way for drag reduction, which is also the goal for the aircraft designers to make a concerted effort to solve[5] [6].

By the long-term observation of the large birds (such as eagles and falcons), it is discovered that they deflect their wings upward in flight to reduce the drag for gliding farther. The vortex was formed at the wing tip and resulted in the induced drag. Hindering the formation of wing tip vortex or destroying the structure of the vortex are the fundamental way to reduce the induced drag. So wing tip drag-reducing device such as cut tip, wing tip sails[7], wing tip winglet were invented. Currently, 
equipped winglets on large transport aircraft in the civilian has been concern widely by the airplane designers. It has become a necessary equipment of the new airplanes. Many countries put into a lot of manpower and financial resources to conduct relevant researches.

\section{Numerical simulation of basic wing}

\section{The wing modeling}

The software Pro/ ENGINEER is used for Wing modeling, and this software are widely used in aviation, aerospace, automotive and other fields by engineering design personnel. At present in the industrial product design, mold design, manufacturing and engineering analysis has been applied[8] [9].

The coordinates of wing root, the turning point and wing tip have been determined with standard RAE2822 supercritical airfoil coordinate basis. The airfoil section has been established. The leading edge sweep angle, semi span dimensions and root tip ratio used to be determined referencing other geometric elements. The surface model is established by using $\mathrm{PRO} / \mathrm{E}$ as shown in Figure 1, Figure 2.

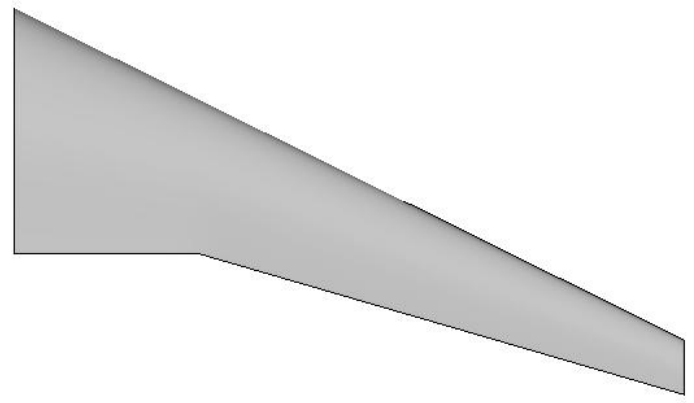

Fig.1 The wing planform

Fig.2 The wing left view

The chord length of the wing root is $C_{t}=0.205 \mathrm{~m}$, the chord length of the turning point is $C_{b p}=0.128 \mathrm{~m}$, the chord length of the wing tip is $C_{t}=0.0458 \mathrm{~m}$, the semi span length is $b / 2=0.553 \mathrm{~m}$. And the leading edge sweep angle $\chi=27^{\circ}$. After the surface mesh model generation, through the display tool to check, the display vertical and horizontal grid line are smooth and continuous. The surface also is smooth and good smoothness. So it can be used for numerical simulation[10].

\section{Discrete calculation region}

In this paper, the discrete software of company Pointwise is used to discretize the model. The software can generate multi block grid structured, unstructured grid and hybrid grid. As one of the world top mesh generation software[11] [12], the discrete software of company Pointwise can import some mainstream CAD (such as Pro/ENGINER, CATIA software etc.). The model file though the software generation, can be seamless connection directly and can use for mesh generation. It has excellent surface repair ability which lays the foundation for the grid generation and provide a convenient. The grid though software generation can be used to present most popular CFD software, the finite element software. It also supports the multiple software data format and boundary definition. On the mesh generation, the density of the mesh and distribution structure are overall considered. And physical changes adapt the simulated physical problems.

The accuracy of calculation results is as far as possible to ensure, at the same time, as far as possible to save computational resources. Because the calculation is a high 
speed compressible problem, in order to ensure the precision, at the leading edge and the trailing edge of the wing it is encrypted necessary. According to the boundary layer thickness, the boundary layer grid is encrypted. In order to save the computing resources, grid computing in a half wing model is selected. The symmetrical boundary conditions is adopted on the symmetric plane[13].

In the discretization process, the surface of the wing is firstly meshing grid, as shown in Figure 3 and Figure 4. In order to ensure that affect of the calculation accuracy.

The C type grid topology of discrete model is used (grid node number 1100,000), as shown in Figure5. Because the computation is compressible problem, the pressure far field boundary conditions(pressure-far-field) is selected on simulation, which is used to describe the infinite freedom compressible flow. The flow calculation region is along the chord direction from the 30 times front chord to 30 times back chord, chord length, and is 10 times span along the spanwise.

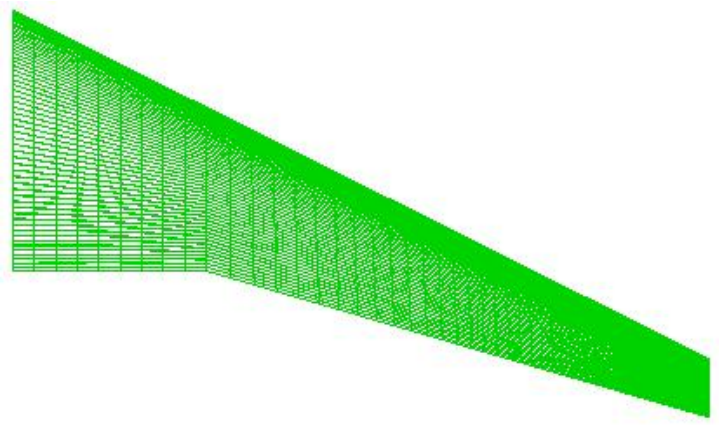

Fig.3 The wing surface grid

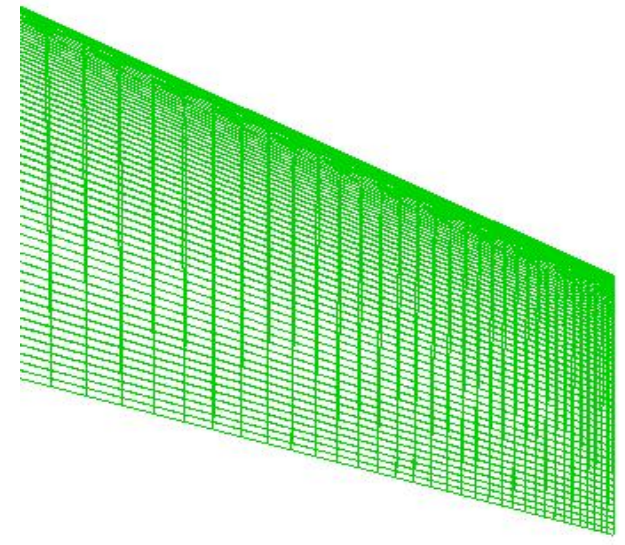

Fig.4 Wing enlarged surface lattice

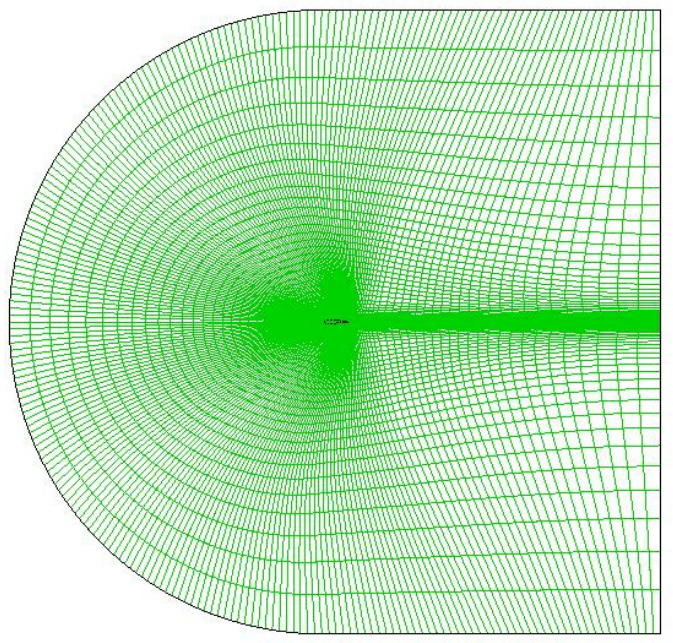

Fig.5 C type grid topology 


\section{Conclusions}

Comparing the two results of calculation, the aerodynamic characteristics and the drag-reducing mechanism of wing with winglet were analyzed in this thesis. Blocking air flow upward, dissipating energy of wing tip vortex, reducing downwash angle and downwash velocity within entire wing span, are the main reasons of winglet drag reduction.

With very high practical value, the next subject should be more in-depth study.

\section{References}

[1]Rumsey CL, Rivers SM, et al. Study of CFD Variation on Transport Configurations from the Second Drag-prediction Workshop[R]. AIAA 2004-0394.

[2]Reneaux J. Overview on drag reduction technologies for civil transport aircraft. European Congress on Computational Methods in Applied Sciences and Engineering[R]. ECCOMAS 2004

[3] Hua Xin. Numerical Simulation And Aerodynamic Performance Comparison Between Seagull Aerofoil and NACA 4412 Aerofoil under Low-Reynolds[J]. Advances in Natural Science, 2010,3 (2) : 244-250.

[4 ] L. V. Schmidt, R. W. Duren. Wing Induced Drag[R]. AIAA 2002-4878.

[5] Hua Xin. High Altitide and Long Endurance Unmaned Aerial Vehicle Bionic Airfoil Research

[6]van Dam, C P. Induced-Drag Characteristics of Crescent-Moon-Shaped Wings[J]. Journal of Aircraft . 1987,24.

[7] JAMSON A. Aerodynamics design via control theory [J].Journal of scientific computing, 1998,3:233-260.

[8]BLACKWELL J. Numerical method to calculate the induced drag or optimal span loading for arbitrary non-planar aircraft [R]. NASA SP-405,May 1976,49-70.

[9]Edward N.Tinoco, Venkatkrishnan. Structured and Unstructured Solvers for the 3rd CFD Drag Prediction Workshop[R].45th AIAA Aerospace Sciences Meeting and Exhibit,2007-255.

[10] Hua Xin. Base on imitation seagullairfoil UVA wing numerical simulation[J]2012,3(1)323-325.

[11] Mark D . Maughmer. The design of winglets for high-performance airplanes[R] .AIAA Paper 2001-2406, June 2001.

[12] van Dam, C P. Efficiency characteristics of crescent-shaped wings and caudal fins[J] , Journal of Aircraft . 1987,325: 435-437.

[13]John C. Vassberg, Edward N.Tinoco „Mori Mani. Summary of Third AIAA CFD Drag Predicion Workshop[J]. 45th AIAA Aerospace Sciences Meeting and Exhibit, 2007-260. 